Medical Expenditures Panel Survey. J Womens Health (Larchmt). 2013. Nov. № 22(11). P. 959-65. doi: 10.1089/jwh.2013.4288.

5. Yu L, Janga B, McAlister R, Jeffe DB, Sonn T. Disparities in Treatment Decisions for Abnormal Uterine Bleeding. J Womens Health (Larchmt). 2021 Feb 12. doi: 10.1089/jwh.2020.8800.

DOI https://doi.org/10.30525/978-9934-26-038-4-39

\title{
ANALYSIS OF CAUSES AND MORTALITY IN ACUTE PANCREATITIS
}

\author{
Formanchuk T. V. \\ Ph. D. in Medicine, Associate Professor, \\ Associate Professor at the Surgery Department № 2 with the course \\ "Fundamentals of Dentistry» \\ National Pirogov Memorial Medical University \\ Vinnytsya, Ukraine
}

Introduction. Acute pancreatitis remains an urgent problem of modern medicine. There is an increase in the incidence of acute pancreatitis worldwide. Despite current trends in multidisciplinary management of this pathology with the involvement of surgeons, gastroenterologists, resuscitators, endoscopists and other specialists, the mortality rate for this pathology remains high and ranges from $1 \%$ to $6,7 \%$. Since the 1970 s, there has been no decrease in mortality of acute pancreatitis $[1,2,3,4,5]$. Mortality of acute pancreatitis occurs from both early and late (septic) groups of complications.

The aim of the work. Analysis of the causes of mortality of acute pancreatitis.

Materials and methods. 48 fatal cases of the patients who were hospitalized in the Vinnytsya emergency hospital with a diagnosis of acute pancreatitis from 2015 to 2020 were analyzed. The diagnosis of acute pancreatitis was formed according to the Atlanta classification, 2012, in the presence of two of any three criteria: typical abdominal pain, serum lipase / amylase values in three times the upper limit of normal, typical signs in the imaging by ultrasound or computer tomography. Complications of acute pancreatitis were diagnosed on the basis of complaints, objective examination data, laboratory, endoscopic and histological examinations, ultrasound diagnostics and computed tomography data.

The youngest patient was 25 years old, the oldest -84 years old. There were 20 women $(41,7 \%)$ and 28 men $(58,3 \%)$. Among 48 fatal cases, 47 
$(97,9 \%)$ developed a necrotic form of acute pancreatitis, and $1(2,1 \%)$ patient had an edematous form. The severity of acute pancreatitis was determined by the APACHE II scale. In all patients was a sever form of acute pancreatitis.

The time from the onset of the disease to the time of admission to the hospital undoubtedly affected the course of the disease and the development of complications of acute pancreatitis and was up to 6 hours in $9(18,75 \%)$ patients, 6-24 hours - in $12(25,0 \%)$ patients , 24-48 hours - in $12(25,0 \%)$ patients and later 48 hours - in $9(18,75 \%)$ patients.

Among the causes of acute pancreatitis in $21(43,7 \%)$ cases was the alimentary factor, in $18(37,5 \%)$ patients - alcohol abuse, unknown etiology in $5(10,4 \%)$ patients, biliary genesis was the cause acute pancreatitis in 2 $(4,2 \%)$ patients, acute pancreatitis after medication - in $1(2,1 \%)$ patient, postoperative pancreatitis developed in $1(2,1 \%)$ patient and was characterized by difficulty of diagnosis due to not clear clinical picture on the background of multimodal analgesia in the early postoperative period.

The hospital-stay length ranged from 12 hours in the lightning form of acute pancreatitis to 83 days in a patient with infectious complications of necrotic pancreatitis.

Results and discussion. Alimentary, alcoholic and biliary factors dominated among the causes of acute pancreatitis.

The distribution of mortality by age revealed that the most of number of deaths occurred in the age group from 60 to 69 years $-11(22,9 \%)$ patients, the lowest number from 20 to 29 years $-2(4,2 \%)$ cases.

In the structure of concomitant diseases, $30(62,5 \%)$ patients had concomitant cardiovascular pathology, $7(14,6 \%)$ patients were obese, and $7(14,6 \%)$ patients had diabetes mellitus. Among the 48 fatal cases, $15(31,25 \%)$ patients were operated.

Among 47 cases of acute necrotic pancreatitis, 34 (72,3\%) foci of necrotic pancreatitis were sterile. $13(27,7 \%)$ patients had infected foci of pancreatic necrosis.

In all cases, there were complications of acute pancreatitis. The structure of complications of acute pancreatitis was dominated by mortality from early complications, which was accompanied by the early development of hemodynamic instability and multiorgan failure. Early complications of acute pancreatitis developed in $23(47,9 \%)$ patients, late complications - in 25 $(52,1 \%)$ patients. Among the most common complications were: endogenous intoxication, respiratory distress syndrome, enzymatic peritonitis, enzymatic pleuritis, multiorgan failure syndrome. Among organ dysfunctions the respiratory system, excretory system, cardiovascular system most often suffered. Among the late complications prevailed: phlegmon of the retroperitoneal space, purulent paracolitis, purulent peritonitis, purulent omentobursitis. 
Conclusions. The main reasons for the development of severe acute pancreatitis, which led to fatal consequences were alcohol abuse, alimentary and biliary factors. In most cases, which ended fataly, there was a late hospitalization of patients. Among all fatal cases in $47(97,9 \%)$ cases there was a destructive form of acute pancreatitis. Mortality of early complications predominated mortality of late complications in acute pancreatitis.

\section{References:}

1. Knudsen J.S., Heide-Jorgensen U., Mortensen F.V., Sorensen H.T., Ehrenstein V. Acute pancreatitis: 31-Year trends in incidence and mortality - a Danish population-based cohort study. Pancreatology. 2020. Vol. 20(7). P. 1332-1339.

2. Pu W.J., Luo G., Chen T., Jing L.H., Hu Q., Li X.Y.,Xia H.F., Deng M.M., Lu M.H., Chen X. A 5-year retrospective cohort study epidemiology, etiology, severity, and outcomes of acute pancreatitis. Pancreas. 2020. Vol. 49 (9). P. 1161-1167.

3. Goldacre M.J., Roberts S.E. Hospital admission for acute pancreatitis in an English population, 1963-98: database study of incidence and mortality. British medical journal. Vol. 328 (7454). P. 1466-1469.

4. Greenberg J.A., Hsu J., Bawazeer M., Marshall J., Friedrich J.O., Nathens A., Coburn N., May G.R., Pearsall E., McLeod R.S. Clinical practice guideline: management of acute pancreatitis. Canadian journal of surgery. 2016. Vol. 59 (2). P. 128-140.

5. Nesvaderani M., Eslick G.D.,Vagg D., Faraj S.,Cox M.R. Epidemiology, aetiology and outcomes of acute pancreatitis: A retrospective cohort study. International journal of surgery. 2015. Vol. 23. P. 68-74. 